\title{
Endoscopic Sclerotherapy with Aluminum Potassium Sulfate and Tannic Acid: An Effective and Less Invasive Strategy for Internal Hemorrhoids
}

\author{
Naoki Muguruma and Tetsuji Takayama \\ Department of Gastroenterology and Oncology, Tokushima University Graduate School of Biomedical Sciences, Tokushima, Japan
}

See "Effectiveness of Endoscopic Sclerotherapy with Aluminum Potassium Sulfate and Tannic Acid as a Non-Surgical Treatment for Internal Hemorrhoids" by Yuichi Tomiki, Jun Aoki, Shunsuke Motegi, et al., on page 581-587.

Internal hemorrhoids are one of the most commonly diagnosed benign anorectal disorders worldwide. They are treated, in part, using suppositories or ointment application in conjunction with preventing straining while defecating. However, additional intervention is required when symptoms become aggravated. In Japan, conventional hemorrhoidectomy has been widely applied for correcting prolapsed internal hemorrhoids. Unfortunately, it is accompanied by postoperative pain and hospitalization, requiring absence from the work place for 2-3 weeks. Recently, several alternative treatment options have become available for patients who do not respond to conservative medical management. Rubber band ligation and sclerotherapy have been shown to be simple and safe treatments for hemorrhoids. The most common sclerosing agent, 5\% phenol almond oil, has been considered ineffective, so an aluminum potassium sulfate and tannic acid (ALTA) combination has been developed in Japan by adjusting certain constituents of the Xiaozhiling, a sclerosing agent developed by Shi in the

Received: August 13, 2019 Accepted: September 9, 2019

Correspondence: Naoki Muguruma

Department of Gastroenterology and Oncology, Tokushima University Graduate School of Biomedical Sciences, 3-18-15, Kuramoto-cho, Tokushima 770-8503, Japan

Tel: +81-88-633-7124, Fax: +81-88-633-9235, E-mail: muguruma.clin.med@ gmail.com

ORCID: https://orcid.org/0000-0002-4740-4809

cc This is an Open Access article distributed under the terms of the Creative Commons Attribution Non-Commercial License (http://creativecommons.org/ licenses/by-nc/3.0) which permits unrestricted non-commercial use, distribution, and reproduction in any medium, provided the original work is properly cited. 1970s. ${ }^{1,2}$ This agent induces an inflammatory reaction resulting in fibrosis, which increases support to tissues while preventing recurrence of prolapse and hemorrhage. ${ }^{3}$ An early clinical study conducted in Japan indicated that the rate of resolution for prolapsing hemorrhoids after an ALTA injection exceeded $90 \%$, similar to the prognosis after surgery. ${ }^{4}$ Follow-up studies showed shorter durations of procedure time and hospital stay, a lower frequency of postoperative pain, and a higher rate of prolapse disappearance with ALTA therapy than with ligation, excision, or other procedures. ${ }^{2,5,6}$ The cumulative success rate of ALTA sclerotherapy in a 5-year follow-up study was also satisfactory (78.2\%-89.3\%).

In this issue of Clinical Endoscopy, Tomiki et al. reported that endoscopic ALTA sclerotherapy was equivalent to standard ALTA therapy in terms of efficacy, adverse events, and recurrence of hemorrhoids. ${ }^{7}$ In this retrospective study, they compared two groups with regard to therapeutic effects, patient satisfaction, adverse events such as fever and anal pain, and the recurrence rate during a 1-year follow-up period. One month after the procedures, $97.0 \%$ of patients in the standard ALTA group experienced therapeutic effects ranging from improvement to cure, and $95.8 \%$ of patients in the endoscopic ALTA group experienced therapeutic effects. The patients reported good satisfaction (very satisfied or satisfied) at all the same time-points in the standard (93.9\%) and endoscopic (95.8\%) ALTA groups. Hemorrhoids recurred in two patients (6.3\%) from the standard ALTA group and in four (8.3\%) from the endoscopic ALTA group. Adverse events occurred 
in four patients (12.1\%) in the standard ALTA group and in six $(12.5 \%)$ in the endoscopic ALTA group. Among these measures, there were no statistically significant differences between the two groups. The injection dose was $21.9 \pm 7.2 \mathrm{~mL}$ in the standard ALTA group and $17.8 \pm 3.4 \mathrm{~mL}$ in the endoscopic ALTA group, which was significantly different $(p<0.01)$.

In the present study, the authors performed a careful, thorough standard vs. endoscopic procedure using a lower ALTA dose as a precaution, yet the therapeutic effect was overall satisfactory. It is expected that the ALTA dose can be increased in future while monitoring patients for adverse events. Endoscopic ALTA may be less embarrassing, especially for women, when comparing the left lateral decubitus position with Sim's position. Using a flexible endoscope is also beneficial when performing a standardized 4-step injection procedure because the visual field is clearly displayed as a high definition image and a precise injection can be accomplished by fine positioning of the scope. Along with further development of endoscopic ALTA, additional research including a multi-center, prospective randomized study with a large number of cases must be conducted. Currently, it is reasonable to conclude that endoscopic ALTA is a less invasive and comparably efficacious therapeutic strategy for hemorrhoids, which should make a large market diffusion for this option.
Conflicts of Interest

The authors have no financial conflicts of interest.

ORCID

Tetsuji Takayama: https://orcid.org/0000-0002-0175-1573

\section{REFERENCES}

1. Hachiro Y, Kunimoto M, Abe T, Kitada M, Ebisawa Y. Aluminum potassium sulfate and tannic acid (ALTA) injection as the mainstay of treatment for internal hemorrhoids. Surg Today 2011;41:806-809.

2. Tokunaga Y, Sasaki H. Impact of less invasive treatments including sclerotherapy with a new agent and hemorrhoidopexy for prolapsing internal hemorrhoids. Int Surg 2013;98:210-213.

3. ASGE Technology Committee, Siddiqui UD, Barth BA, et al. Devices for the endoscopic treatment of hemorrhoids. Gastrointest Endosc 2014;79:8-14.

4. Hachiro Y, Kunimoto M, Abe T, Kusano M. A new sclerosing therapy for internal hemorrhoids: experience in 200 cases with Zione injection. Journal of the Japan Society of Colo-Proctology 2006;59:317-321.

5. Tomiki Y, Ono S, Aoki J, et al. Treatment of internal hemorrhoids by endoscopic sclerotherapy with aluminum potassium sulfate and tannic acid. Diagn Ther Endosc 2015;2015:517690.

6. Miyamoto H, Hada T, Ishiyama G, Ono $\mathrm{Y}$, Watanabe H. Aluminum potassium sulfate and tannic acid sclerotherapy for Goligher grades II and III hemorrhoids: results from a multicenter study. World J Hepatol 2016;8:844-849.

7. Tomiki Y, Aoki J, Motegi S, et al. Effectiveness of endoscopic sclerotherapy with aluminum potassium sulfate and tannic acid as a non-surgical treatment for internal hemorrhoids. Clin Endosc 2019;52:581-587. 